\title{
Tipologías textuales: de Aristóteles a la clasificación múltiple
}

\author{
Marcela A. Amaya García*
}

\begin{abstract}
Resumen
Este trabajo se centra en la revisión crítica de los principales modelos tipológicos destinados a la clasificación de los textos, teniendo por objetivos identificar las diferencias entre unos y otros, dar cuenta de la evolución en los criterios utilizados para la clasificación textual y entregar una aproximación conceptual de cada clase o tipo de texto. El análisis de los planteamientos referidos a la determinación de los tipos de textos muestra cómo se trata un ámbito de la lingüística que ha cobrado especial impulso a partir de los años setenta, considerándose tipologías disímiles para resolver la complejidad del texto.
\end{abstract}

Palabras clave: Clase textual - tipo de texto - tipología textual - criterios de clasificación textual

\begin{abstract}
This research in centered on the review of the principal tipological models destined to the classification of the texts, begin for objetive identify the difference between some and others. Show the evolution in the criteria used for the textual classification and delivered a conceptual approximation of class and tipe of texts. The analysis of the approches recounted to the determination of tipes of texts show how it is, and question an area of the linguistics that area has resived and special impulse from the seventies begin considered dissimilar to solve the complexity of the text.
\end{abstract}

Keywords: Textual class, tipe of texts, textual typology, criteria of texual classification

Periodista, Magíster en Lingüística Hispánica. Universidad Católica Silva Henríquez. mamaya@ucsh.cl 
Tipologías textuales: de Aristóteles a la clasificación múltiple / Marcela Amaya García

\section{Introducción}

Mientras autores como Bajtín (2002), Ciapuscio (1994, 2005), Calsamiglia y Tusón (1999), Loureda (2003), Charadeau (2004) y Simón (2006) han proporcionado importantes aportes teóricos relativos al texto y sus criterios de clasificación; otros han puesto sus esfuerzos en un acercamiento a la problemática de los géneros discursivos desde una perspectiva más bien pragmática, por ejemplo, Moyano (2001), quien construye una propuesta de clasificación de géneros científicos de mayor circulación en la Argentina.

Siguiendo en la línea de los estudios teórico-prácticos, cabe destacar las investigaciones desarrolladas desde el enfoque de la lingüística funcional, específicamente, en torno a la relación género-registro realizados por Eggins y Martin (2003) y Suau (2000), así como el trabajo elaborado por Parodi y Gramajo (2003) en el marco del acercamiento a un discurso especializado según criterios de multiniveles.

Aun cuando los estudios mencionados en los párrafos precedentes han buscado contribuir a la comprensión de la cuestión de las tipologías textuales, lo cierto es que todavía persiste un vacío en la sistematización y revisión crítica del conocimiento acumulado relativo a esta materia.

Como una aproximación inicial para salvar dicha brecha surge el presente trabajo, cuya primera parte presenta algunos aspectos teóricos en función del concepto de clase textual o tipo textual. Posteriormente, se plantean y comentan las teorías tipológicas más representativas que datan desde Aristóteles hasta estos días agrupadas por criterios lingüísticos, extralingüísticos, entre otros. Finalmente, en las conclusiones se hace una síntesis de los principales hallazgos y se pone en perspectiva la realización de futuras investigaciones.

\section{Metodología}

La estrategia metodológica utilizada en este estudio corresponde a investigación documental en tanto técnica de obtención de información. Al respecto, Valles (1996), evocando a Ruiz Olabuénaga e Ispizua (1989), señala que ésta forma 
parte de las técnicas aplicables a estudios cualitativos, donde la lectura de textos permite una revisión exhaustiva de distintos documentos.

A la observación y la entrevista podemos añadir una tercera técnica de recolección de datos, la lectura de textos, entendiendo tales, todos los documentos que contienen significado (una carta, un periódico, una autobiografía, una estatua, un edificio, las pinturas de una cueva prehistórica, las tumbas faraónicas...).

A todos estos 'textos', en realidad, se les puede 'entrevistar' mediante preguntas implícitas y se les puede 'observar' con la misma intensidad y emoción con la que se observa un rito nupcial, una pelea callejera, una manifestación popular. En este caso la lectura es una mezcla de entrevista/observación y puede desarrollarse como cualquiera de ellas. (Valles, 1996: 120).

En lo particular, esta investigación se centra -de acuerdo a la clasificación del autor antes citado- en el análisis de datos secundarios, es decir, la revisión crítica de una amplia gama de escritos (detallados en las referencias bibliográficas) referidos a los principales modelos tipológicos.

[Se consideró] un cúmulo de informaciones que se hallan recogidas o publicadas por diversas instituciones sin propósitos específicos deinvestigación social, sino con otros fines muy variados, fundamentalmente, proveer de información o documentación a los órganos del Estado o al público. Entra aquí un abigarrado conjunto de datos censales, literatura, política, biografías, ficheros de distintas instituciones, memorias, publicaciones periódicas. (Valles, 1996: 121-122).

En este punto resulta importante precisar la aproximación conceptual que Valles (1996) presenta en relación al término documento. En primer lugar, el autor, tomando la definición Earlandson et al., (1993) explica que este vocablo engloba una variada y heterogénea gama de registros, tanto escritos como simbólicos. En segundo lugar, a partir de MacDonald y Tipton (1993), Valles señala que un documento tiene las siguientes características intrínsecas: son escritos, por lo tanto, son posibles de leer y tienen como intención dar cuenta de un aspecto del mundo social.

Por último, la elección de esta técnica de recogida de información se funda en las ventajas que Valles (1996: 129) describe, citando a Webb y otros (1966) y Hodder (1994).

V1-3. Bajo coste de una gran cantidad de material informativo, que en algunos casos tiene un carácter periódico (los medios de comunicación por ejemplo, o el Diario de Sesiones de la Cortes).

V4. No reactividad: A diferencia de la información obtenida directamente por el investigador, mediante técnicas de observación o 
conversación (cuestionarios, entrevistas), el material documental suele producirse en contextos naturales de interacción social. Esto significa que, debido a la ausencia del investigador, no habrá que preocuparse por las reacciones que éste puede provocar en las personas cuando se saben investigadas. Claro otras fuentes de reactividad (o de otro tipo) sí pueden haber contaminado ya la información contenida en los documentos. Pero estos son problemas que se detectan y resuelven en la evaluación e interpretación de la documentación misma (...).

V5. Exclusividad. El contenido informativo que proporcionan algunos materiales documentales tiene un cierto carácter único, pues difiere del que puede obtenerse mediante las técnicas directas de observación y conversación.

V6. Historicidad. Los escritos, las imágenes y las voces grabadas permanecen en el tiempo, si alguien las conserva o archiva (...).

\section{Hacia una aproximación conceptual de clase o tipo textual}

Si bien cuando se evocan los vocablos "tipología textual", "clase textual" o "tipo de texto" el sentido común indica como primer referente una probable categorización en la que se enmarcarían los escritos; lo claro es que en relación a dicho concepto se ha desarrollado una vasta discusión teórica que, seguramente, está lejos de finalizar.

Tanto Ciaspucio (1994), Loureda (2003) como Calsamiglia y Tusón (1999) reconocen en Aristóteles al primero en aventurarse en una propuesta de clasificación textual -no conceptual- compuesta por tres géneros: demostrativo (epidíctico o de ocasión), judicial (legal o forense) y deliberativo (políticos).

Al respecto, Calsamiglia y Tusón (1999) precisan que "Aristóteles aborda su clasificación atendiendo a los discurso en uso en la vida social pública de su tiempo: orales y ante una audiencia y, por lo tanto, se refiere a ámbitos de la vida institucional" (253). Más allá de esta aclaración, Loureda (2003) resume la importancia de la perspectiva aristotélica como sigue:"(...) La retórica aporta dos grandes referentes a la tipología textual: el primero, una clasificación de los géneros; y el segundo, un modo de entender los textos" (55).

Sin embargo, a juicio de Ciapuscio (1994),"M. Bajtín, desde una perspectiva semiótica-social, ha realizado uno de los mayores aportes al estudio de la temática al introducir el concepto de género discursivo en tanto conjunto de enunciados relativamente estable ligado a una esfera social determinada" (14).

La teoría bajtiniana, en efecto, puede considerarse como la pionera en reflexionar de manera formal acerca de la problemática de los géneros, plan- 
teando que "todo estilo está indisolublemente vinculado con el enunciado y con las formas típicas de enunciados, es decir, con los géneros discursivos" y que "todo enunciado, oral o escrito, primario o secundario, en cualquier esfera de la comunicación discursiva, es individual y por lo tanto puede reflejar la individualidad del hablante (o del escritor), es decir, puede poseer un estilo individual" (Bajtín, 2002: 251). Asimismo, complementando la teoría de la enunciación, el autor establece la taxonomía de los géneros primarios o simples, que se dan en la situación comunicativa inmediata y, los géneros secundarios o complejos, que requieren mayor elaboración y se dan en situaciones de comunicación mediada.

Calsamiglia y Tusón (1999), citando los postulados de Bajtín (1979), agregan que "(...) lo que condiciona la existencia de un determinado género son cuatro factores: los temas, la estructura interna, el registro (o estilo funcional) utilizado y la relativa estabilidad de todo ello" (257). "Los tres momentos mencionados -el contenido temático, el estilo y la composición- están vinculados indisolublemente en la totalidad del enunciado y se determinan, de modo semejante, por la especificidad de una esfera dada de comunicación" (Bajtín, 2002: 248).

Por otra parte, según Charadeau y Maingueneau (2005) "el discurso (o texto) es una noción que se encontraba ya en la filosofía clásica, donde al conocimiento discursivo por concatenación de razones se le oponía el conocimiento intuitivo. Su valor estaba entonces próximo al logos griego. En lingüística, esta noción puesta en primer plano por G. Guillaume experimentó un desarrollo fulgurante con la declinación del estructuralismo y el ascenso de las corrientes pragmáticas" (179).

Concuerda con este planteamiento Ciaspucio (1994), quien afirma:

La irrupción y el auge de la pragmática, el convencimiento científico general acerca de la necesidad de los estudios interdisciplinarios así como la tendencia a la ampliación de los objetos de estudio en las ciencias sociales y humanas favorecieron el interés por las tipologías textuales, desde distintas perspectivas, con diferentes herramientas y objetivos (15).

Cabe señalar que la autora citada, adscribiendo a la lingüística del texto, utiliza indistintamente los términos clase textual y tipo textual. De manera que entiende el primero como una "clasificación de tipo empírica" y, el segundo, como una "categoría ligada a una teoría para la clasificación científica de los textos" (Ciaspucio, 1994: 25).

De acuerdo a Álvarez (2001: 11-12) existe una polisemia entre los términos texto y discurso, lo que implica un uso ambiguo de ambos. Sin embargo, precisa que mientras "el texto es una configuración lingüística. Es un conjunto de elementos lingüísticos (palabras, oraciones...) organizados según reglas estrictas; el discurso es la emisión concreta de un texto, por un enunciado determinado, en una situación de comunicación determinada". "Todo esto hace que exista 
Tipologías textuales: de Aristóteles a la clasificación múltiple / Marcela Amaya García

una extrema imprecisión terminológica cuando se pretende caracterizar los diversos tipos de textos". Básicamente, su clasificación se basa en "tres criterios fundamentales" (Álvarez: 2001: 144-146).

1. Un criterio sociocultural permite distinguir tipos de textos que son productos de las prácticas discursivas de una sociedad.

2. Un criterio funcional permite caracterizar "tipo de discursos" (...) según la función que estos cumplen en la comunicación. Es decir, según la relación que el sujeto comunicante establece con su interlocutor en términos de su proyecto de habla.

3. Un criterio estructural (o construccional), por último, permite caracterizar los textos según el modo de organización dominante.

Calsamiglia y Tusón (1999), también, se refieren a la imprecisión conceptual de género, diciendo que "si bien es un concepto sujeto a controversia y a veces difícil de delimitar, su uso se ha extendido para clasificar gran parte de los productos culturales en los que el uso de la palabra es un elemento fundamental. Actualmente se aplica a las manifestaciones que han generado las nuevas tecnologías de la comunicación y en especial al cine, la radio y la televisión" (252). Villalobos (1997) discrepa de la anterior idea, ya que cuestiona la necesidad de efectuar clasificaciones tipológicas en el campo de las comunicaciones, en especial, del periodismo: "(...) ¿Realmente es posible clasificar este discurso que es tan mutante en la sociedad actual? ¿Y si fuera posible cuál sería la utilidad del encasillamiento" (8-9).

Aunque De Beaugrande y Dressler (1986) no elaboran una taxonomía textual, sí proponen siete principios que todo texto debiese cumplir para ser considerado como comunicativo. En pocas palabras, estos son: la cohesión, la coherencia, la intencionalidad, la aceptabilidad, la situacionalidad, la intertextualidad y la informatividad.

Loureda (2003), en tanto, describe el texto en función de dos dimensiones: la dimensión universal y la dimensión tradicional. La primera, implica la presencia de características comunes a todos los tipos de textos y, la segunda, encierra la existencia de rasgos propios de un género. Al respecto, el autor recalca que "(...) los tipos de texto son herencias culturales que solucionan las necesidades expresivas y comunicativas de los hablantes. Funcionan, como hecho pragmático, en la esfera del hablante y en la del oyente: al primero le imponen restricciones de codificación; al segundo le sirven de guía para la interpretación" (32). A su vez, señala que los tipos de textos presentan rasgos esenciales, es decir, que lo definen como tal y, concomitantes, que ayudan a configurar el tipo de texto, pero no lo crean. Por último, sobre los géneros discursos Loureda (2003) concluye que son "(...) modelos ideales intuitivos aglutinadores paradigmáticos de los caracteres necesarios de todos los textos de una misma naturaleza" (37). 
En síntesis, a la luz de lo expuesto en este apartado y citando a Simón (2006) se entiende:"[...] Que se debe seguir trabajando en el ámbito de la lingüística del texto, debido a que el seno de la misma persisten asuntos sin resolver del todo, como la conceptualización de texto y tipologías textuales" (161).

\section{Criterios de clasificación textual: principales modelos tipológicos}

La necesidad de clasificar es intrínseca al ser humano: para comprender el mundo que nos rodea, percibimos las similitudes y diferencias y a partir de allí surge inmediatamente la necesidad de ordenar, jerarquizar, en suma, de establecer tipos de objetos, acciones, eventos, situaciones sobre la base de criterios de orden diverso. Esta forma de operar del sistema cognitivo humano tuvo desde la antigüedad su reflejo en las artes y humanidades y constituye hoy en día una preocupación fundamental en los estudios discursivos y textuales. La finalidad de la clasificación o tipologización no se dirige en estas disciplinas hacia el mundo externo en general sino hacia las producciones lingüísticas de las personas, esto es, hacia los textos. ¿Cómo distinguimos una factura de una citación judicial?

¿Cómo, un coloquio de una declaración? ¿Cómo dictaminamos sin ningún inconveniente que un texto es una reseña crítica y no una recomendación o un aval? Aunque una vez más pueda parecer que los lingüistas se ocupan de lo obvio, no es tan fácil dar respuesta a estas interrogantes. (Ciaspucio, 1994: 13).

Tal como lo señala Ciaspucio (1994) la idea de entender la complejidad del texto ha sido una inquietud permanente entre los lingüistas, esto es, desde Aristóteles a la actualidad. Así, han nacido distintas clasificaciones o taxonomías tipológicas basadas en criterios disímiles -que han evolucionado en el tiempodestinadas a brindar mayores antecedentes y/o pormenores acerca del universo textual que incluye cartas, noticias, artículos científicos, obituarios, postales, libros escolares, novelas, microcuentos, fotonovelas, etc.

A continuación, siguiendo los planteamientos de Loureda (2003), Ciaspucio (1994, 2005), Charadeau (2004) y Eggins y Martin (2003), entre otros, se presentan y revisan de manera didáctica los principales modelos de categorización textual organizados en función de criterios propuestos por los teóricos mencionados con anterioridad.

\subsection{Tipologías que parten de características verbales}

Este tipo de clasificaciones -influenciadas por las corrientes estructuralistas- ponen énfasis en las características internas de los textos, tales como la estructuración del contenido, las temáticas abordadas, las marcas modales y 
Tipologías textuales: de Aristóteles a la clasificación múltiple / Marcela Amaya García

las referencias o correferencias, etc., centrándose específicamente en los rasgos aspectuales de los verbos. Vale decir, se trata de una aproximación microestructural de carácter morfosintáctico.

Se incluyen en este ítem las tipologías de Weinrich, Biber $(1985,1986)$, Posner y Gülich (1986).

\subsubsection{Weinrich}

Mediante el análisis morfosintáctico pretende establecer la combinación de esta clase de componentes específica de cada género. El punto central es el verbo a partir de lo cual formular 14 propiedades, aplicando pares de oposiciones.

$\begin{array}{llll}\text { Ejemplo: } & \text { Forma activa (entiende) } & - & \text { Forma pasiva (se entiende) } \\ & \text { Singular (caminó) } & - & \text { Plural (caminaron) }\end{array}$

\subsubsection{Biber (1985, 1986)}

Su trabajo se basa en el análisis de cómo se comportan sesenta y siete rasgos lingüísticos en un corpus de 481 textos que representan veintitrés géneros de uso generalizado en el habla inglesa, por ejemplo, reportaje, biografías, etc. A partir de ello, establece -según Loureda (2003) - cinco dimensiones de variación, dando origen a una caracterización empírica de ocho tipos de texto: dos de carácter interactivo (interacción íntima interpersonal y interacción informativa); tres de índole expositiva (exposición científica, exposición académica y exposición narrativa general); dos de origen narrativo (exposición narrativa y narración imaginativa), además, se contempla el reportaje contextualizado y la persuasión subjetiva.

\subsubsection{Posner}

Clasifica los géneros a partir de sus estructuras temáticas, es decir, estudia las relaciones léxico-semánticas con los temas del texto, ya sea principal, secundario, etc.

\subsubsection{Gülich (1986)}

Presenta una visión lingüística-textual de orientación empírica, o sea, rastrea en textos orales y escritos aquellas expresiones lingüísticas que de alguna manera señalan pertenencia a ciertas clases textuales. Sus aportes se resumen en que su propuesta trasciende el nivel puramente teórico para conocer el texto en la cotidianidad e incorpora por primera vez el discurso oral.

\subsection{Tipologías que tienen en cuenta características pragmáticas}

Estas clasificaciones conciben el texto como un hecho pragmático, por lo tanto, el análisis desde este punto de vista contempla la intención del hablante, la relación 
entre los interlocutores, la condición del oyente y la situación comunicativa y/o contexto en que está inmerso el discurso, en especial, el acto de habla.

Se destacan entre las tipologías pertenecientes a esta corriente las de Sandig (1972), Werlich (1975), Grosse (1976) y Adam (1991).

\subsubsection{Sanding (1972)}

Presenta una caracterización de los textos de carácter binaria (positivo-negativo) a partir de rasgos pragmáticos y verbales. En el fondo, la autora construye un esquema de rasgos arbitrarios -que se presentan en la siguiente tabla a modo de ejemplo- donde deben calzar los distintos textos.

\begin{tabular}{|c|c|c|c|c|c|c|c|c|c|c|c|c|c|c|c|c|c|c|c|c|}
\hline $\begin{array}{l}\text { Rasgos } \\
\text { Tipos de } \\
\text { texto }\end{array}$ & 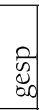 & $\begin{array}{l}\text { है } \\
\text { क } \\
\text { क }\end{array}$ & 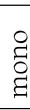 & $\frac{\pi}{\pi}$ & 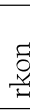 & $\begin{array}{l}5 \\
\frac{2}{N}\end{array}$ & 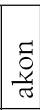 & $\underset{\pi}{\pi}$ & 苞 & $\underset{\tilde{\sigma}}{\stackrel{\Xi}{\Xi}}$ & 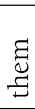 & 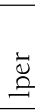 & $\begin{array}{l}\check{\tilde{U}} \\
\text { ते }\end{array}$ & mे & 芯 & 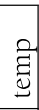 & $\mid \begin{array}{c}\tilde{0} \\
0 \\
\\
: 0\end{array}$ & $\begin{array}{l}\vec{Z} \\
\mathbb{\Psi} \\
\end{array}$ & $\begin{array}{l}\vdots \\
\text { ڤै } \\
\end{array}$ & \begin{tabular}{l}
$\overrightarrow{2}$ \\
\multirow{2}{*}{} \\
\end{tabular} \\
\hline carta & + & \pm & \pm & - & - & - & - & + & + & - & \pm & + & + & + & \pm & \pm & \pm & \pm & + & \pm \\
\hline anuncio & \pm & \pm & \pm & \pm & \pm & \pm & \pm & \pm & \pm & - & \pm & \pm & \pm & \pm & \pm & \pm & \pm & \pm & \pm & - \\
\hline noticia de radio & + & - & + & - & - & + & + & + & + & - & - & - & - & + & - & + & - & + & + & - \\
\hline $\begin{array}{l}\text { conversación } \\
\text { familiar }\end{array}$ & + & + & - & \pm & + & + & + & \pm & - & - & - & + & + & + & \pm & \pm & \pm & + & \pm & + \\
\hline
\end{tabular}

(Loureda, 2003: 62).

\subsubsection{Werlich (1975)}

Es una tipología de carácter cognitivo, pues considera que la secuencia o forma de los textos está estrechamente relacionado con procesos de categorización de la realidad por medio del pensamiento. Incorpora el concepto de secuencia textual, dando lugar a los siguientes tipos de textos:

Narrativos: en ellos se cuenta cómo algo o alguien actúa a través de verbos que indican acciones.

Descriptivos: el proceso cognitivo dominante es la indicación de cómo es algo o alguien. Las secuencias se construyen a partir del verbo ser.

Expositivos: se dice algo de un tema por medio de una estructura verbal que incorpora el verbo ser con un predicado nominal o el verbo tener con un objeto directo.

Instructivos: se indica cómo hacer algo por medio de una estructura verbal de corte imperativa.

\subsubsection{Grosse (1976)}

Utiliza el concepto de función textual que parte del modo en que el hablante (o productor textual) desea que se interprete su texto por parte del lector u 
Tipologías textuales: de Aristóteles a la clasificación múltiple / Marcela Amaya García

oyente. En consecuencia, el autor enumera siete funciones básicas que dan vida a la siguiente clasificación textual:

Normativas: leyes, estatutos, etc.

De contacto: saludos, felicitaciones, etc.

De indicación de grupo: himnos religiosos, lemas, etc.

Poética: poemas, novelas, etc.

De automanifestación: biografías, diarios de vida, etc.

Exhortativa: solicitud, pregunta, etc.

De transferencia de información: noticias, informes meteorológi-

cos, etc.

\subsubsection{Adam (1991)}

Según este autor la función predominante en el texto es clave para determinar su clasificación. En 1985, toma como base la taxonomía de Werlich y agrega a ésta el aspecto conversacional, predictivo y retórico. Sin embargo, en 1992 reconsidera su trabajo y reduce los tipos a cinco: narrativo, descriptivo, argumentativo, explicativo y dialógico/conversacional. Del mismo modo, establece que no existe una clasificación, sino que más bien se trata de formas prototípicas que se mezclan entre sí y que lo fundamental es la estructura típica que adoptan las secuencias al interior del texto.

\subsection{Tipologías que sólo parten de características pragmáticas}

\subsubsection{Glinz}

Parte de la función comunicativa, es decir, del objetivo que se quiere cumplir con el discurso, distinguiendo cinco tipos de textos:

Textos que crean acuerdos: leyes, contratos, etc.

Textos dirigistas: peticiones, manuales, libros de enseñanza, etc.

Textos almacenadores: apuntes, censos, guías de teléfonos, etc.

Textos de comunicación privada: cartas, postales, etc.

Textos descriptivos públicos: noticias, novelas, etc.

\subsection{Tipos textuales según criterios de clasificación múltiples}

\subsubsection{Brinker (1988)}

A partir de la cotidianeidad se interroga por los rasgos que en la vida diaria distinguen clases de textos y, sobre esa base, hacerlos explícitos e interpretarlos desde el punto de vista lingüístico-teórico. Da pie a criterios de clasificación, entendidos como propuestas tentativas en las cuales enmarcar los diversos textos. Estos criterios son: 
Criterio de la función textual: incluyen los textos informativos, de contacto, apelativos, obligativos y declarativos.

Criterios contextuales: dice relación con el medio en que se da y el ámbito de acción del texto.

Criterios estructurales: indican el tipo de tema textual y tipo de despliegue temático del texto, distinguiendo entre descriptivos, narrativos, explicativos y argumentativos.

\subsection{Tipologías de varios niveles de clasificación}

\subsubsection{Heinemann y Vieweger (1991)}

Los autores parten de la base que definir una tipología única es muy difícil $y$, de acuerdo al enfoque procedural al que pertenecen, que concibe que los hablantes tienen un saber para la comprensión de esquemas textuales globales (clases textuales), señalan que este saber se realiza por medio de clasificaciones multidimensionales de representaciones prototípicas a distintos niveles. Los niveles para la tipologización de textos propuestos son los siguientes:

Tipos de función: se refiere a la interacción comunicativa, es decir, efecto que puede tener un texto, por ejemplo, expresarse o liberarse.

Tipos de situación: considera el tiempo, el lugar y el conocimiento sobre esferas comunicativas, entre otros.

Tipos de procedimiento: implica el tipo de procedimiento que los productores textuales han elegido para que la comunicación sea exitosa, tales como qué información hacer presente en el texto.

Tipos de estructuración textual: aquí se contempla la elección de la estructura interna y, los mecanismos de conexión y cohesión.

Esquema de formulaciones prototípicas: los autores establecen que existen modelos de formulación, o sea, palabras o construcciones que se han conservado y fijado como prototípicas de determinadas clases textuales. Por ejemplo: "evaluación" se encontraría presente en los diferentes discursos relativos al ámbito escolar.

\subsubsection{Charadeau (2004)}

Desde una perspectiva psicosocio comunicativa, este autor afirma que una definición de género discursivo implica la articulación entre tres niveles y su respectiva correlación de los elementos componentes de ellos. Los niveles sugeridos son: 
Tipologías textuales: de Aristóteles a la clasificación múltiple / Marcela Amaya García

Situacional de las restricciones: en este punto deben ser considerados los datos externos que sólo nacen cuando se pretende construir un texto o discurso.

De la organización discursiva: es determinada por las instrucciones situacionales.

De las marcas formales: constituyen las ocurrencias formales en los diversos géneros.

\subsection{Perspectiva lingüística funcional}

\subsubsection{Hasan $(1977,1984)$}

Su concepto de género aspira a preservar las categorías de campo, tenor y modo de Halliday (1978). El principal aporte de esta autora está dado por la noción de potencial de estructura genérica, que generaliza la gama de posibilidades de secuenciación asociadas con un género en particular. Para entender este planteamiento, un ejemplo ofrecido es el análisis de una transacción en el mercado (Eggins y Martin, 2003): "Saludos^^ Preguntas sobre el Objeto en Venta^ Investigación del Objeto en Venta^^ Negociación ^ Conclusión” (31).

\subsubsection{Eggins y Martin (2003)}

Estos teóricos, precursores de la Escuela Género de Sydney, proponen un modelo estratificado de contexto que se compone de dos niveles o capas: el género y el registro, donde el primero asume una categoría superior. En otras palabras, el registro y el género son dos planos de realización en una visión semiótica del texto. "El texto es una realización de tipos de contextos como la escenificación de lo que interesa a los miembros de una cultura en situaciones determinadas" (Eggins y Martin: 2003: 31). Destaca entre los trabajos desarrollados bajo esta línea, el de Suau (2000) dirigido al estudio del género y el registro en la traducción del discurso profesional.

Tras esta revisión documentada, como una contribución desde la realidad lingüística nacional al conocimiento y desarrollo de las tipologías textuales, la autora propone la siguiente definición de clase textual:

Agrupación prototípica de rasgos lingüísticos (morfológicos-semánticos) y extralingüísticos (contextuales-situacionales) mínimos que configuran los textos de una misma categoría.

De esta aproximación conceptual se desprenden tres consideraciones:

La existencia de un conglomerado mínimo de características que se presentan en forma recurrente en un mismo tipo de textos. 
En relación a los rasgos lingüísticos, se recoge la idea de que ciertos vocablos (conectores, verbos, etc.) están presentes en general en un mismo tipo de texto.

Por último, incluir un mínimo de rasgos extralingüísticos intenta abarcar el texto en su extensión, puesto que el contexto entendido desde una mirada pragmalingüística mantiene un influjo dialéctico con el discurso. Asimismo, significa identificar funciones textuales y describir situaciones comunicativas en que se dan determinados discursos.

\section{Conclusiones}

La problemática de las tipologías textuales es una inquietud que ha estado presente permanentemente entre los lingüistas. En primera instancia, de manera intuitiva (Aristóteles) hasta llegar a trabajos formales, como es el caso de las clasificaciones múltiples y de multinivel, etc.

No existe consenso entre los expertos respecto a la definición de clase o tipo textual. Por ello, se propende a un uso indistinto y ambiguo de los conceptos.

El mayor impulso de los estudios relativos a la clasificación textual se da a partir de los años '70, cuestión que coincide con la irrupción de las corrientes pragmáticas y la lingüística textual.

Se observa un vacío en los trabajos sobre la temática, desde los planteamientos de Bajtín hasta la aparición de los primeros teóricos que consideraron categorizaciones basados en elementos estrictamente lingüísticos como el verbo.

Los criterios de clasificación textual han evolucionado desde lo particular al estudiar las microestructuras (verbos) a lo general al considerar los distintos aspectos de un texto por medio de las taxonomías que consideran niveles textuales.

Las últimas propuestas teóricas relativas a esta materia intentan dar cuenta de la complejidad del texto de manera amplia y exhaustiva, incluyendo elementos tanto internos como externos.

En la actualidad, se constata la falta de lingüistas expertos en esta materia capaces de concatenar el conocimiento acumulado y formular propuestas teóricas destinadas a incrementar la aproximación a las tipologías textuales.

En vista de lo descrito, esta investigación abre la oportunidad cierta de acercarse a las tipologías textuales no sólo para entender los diversos textos presentes en la cotidianeidad inmediata, sino que también para apoyar y fomentar los niveles de comprensión lectora de los hablantes y/o lectores (adultos y niños) del país. En cualquier caso, es importante señalar que este trabajo constituye una primera aproximación al fenómeno. 
Tipologías textuales: de Aristóteles a la clasificación múltiple / Marcela Amaya García

\section{Bibliografía}

Álvarez, G. (2001). Textos y discursos. Introducción a la Lingüística del Texto. Concepción: Editorial Universidad de Concepción.

Bajtín, M. (2002). Estética de la creación verbal. Argentina: Siglo XXI Editores.

Calsamiglia, H. y Tusón, A. (1999). Las cosas del decir. Manual de análisis del discurso. Barcelona: Ariel Lingüística.

Ciapuscio, G. (1994). Tipos textuales. Buenos Aires: Eudeba.

Ciapuscio, G. (2005). "La noción de género en la lingüística sistémico funcional y en la lingüística textual". Signos 38(57): 31-48. [En línea] Obtenido 02 abril 2010 desde: http://www.scielo.cl/scielo. php?pid=S0718-09342005000100003\&script=sci_arttext

Charadeau, P. (2004). "La problemática de los géneros. De la situación a la construcción textual". Signos 37(56): 23-39. [En línea] Obtenido 02 abril 2010 http://www.scielo.cl/scielo.php?pid=S0718$09342004005600003 \&$ script=sci_arttext

Charadeau, P. y Maingueneau, D. (2005). Diccionario de análisis del discurso. Buenos Aires: Amorrortu.

De Beaugrande, R. y W, Dressler. (1986). Basic Notions. Introduction to Text Linguistics. Londres: Longman.

Eggins, S. y Martin, J. (2003). "El contexto como género: una perspectiva lingüística funcional". Signos 36(54): 185-205. [En línea] Obtenido 02 abril 2010 http://www.scielo.cl/scielo.php?pid=S0718$09342003005400005 \&$ script=sci_arttext

Loureda, O. (2003). Introducción a la tipología textual. Madrid: Arco Libros.

Moyano, E. (2001). "Una clasificación de géneros científicos". XIX Congreso AESLA: 1-9. [En línea] Obtenido 02 abril 2010 http://userpage.fu-berlin. de/vazquez/vazquez/Clasificacion\%20de\%20generos\%20 MOYANO.pdf

Parodi, G. y Gramajo, A. (2003). "Los tipos textuales del corpus técnico profesional PUCV 2003: una aproximación multiniveles". Signos 36(54): 207-223. [En línea] Obtenido 02 abril 2010 http://www.scielo.cl/scielo. php?pid=S0718-09342003005400006\&script=sci_arttext

Simón, J. (2006). "Propuesta para la determinación de los tipos de textos". Sapiens 1:163-179. En línea] Obtenido 02 abril 2010 http://www2. scielo.org.ve/scielo.php?script=sci_abstract\&pid=S1317$58152006000100010 \& \operatorname{lng}=$ pt\&nrm $=$ iso

Suau, F. (2000). "El género y el registro en la traducción de discurso profesional: un enfoque funcional aplicable a cualquier lengua de especialidad". Quaderns de Filología Vol. IV: S/N. [En línea] Obtenido 02 abril 2010 http://www.ub.es/filhis/culturele/tinasuau.html

Valles, M. (1996). Técnicas Cualitativas de Investigación Social. Reflexión metodológica y práctica profesional. Madrid: Editorial Síntesis. 
Literatura y Lingüística Nº22

Villalobos, C. (1997). "El discurso periodístico y la encrucijada de los géneros". Reflexiones 56: S/N. [En línea] Obtenido 02 abril 2010 http:// www.reflexiones.fcs.ucr.ac.cr/edanteriores 12.php 